Born too soon: the global action report on preterm birth. Geneva: World Health Organization, 2012.

2. Waters KM, Stram DO, Hassanein MT, et al. Consistent association of type 2 diabetes risk variants found in Europeans in diverse racial and ethnic groups. PLoS Genet 2010;6(8):e1001078.

3. Liu JZ, van Sommeren S, Huang H, et al. Association analy- ses identify 38 susceptibility loci for inflammatory bowel disease and highlight shared genetic risk across populations. Nat Genet 2015;47:979-86.

4. Romero R, Dey SK, Fisher SJ. Preterm labor: one syndrome, many causes. Science 2014;345:760-5.

DOI: $10.1056 / N E J M c 1713902$

\title{
Third Dose of MMR Vaccine for Mumps Control
}

TO THE EDITOR: From the end of July through September 2017, a total of 26 cases of mumps occurred in a company of 140 military recruits in a single compound. Among all the soldiers on base, $91.4 \%$ had received two or more doses of the measles-mumps-rubella (MMR) vaccine before the outbreak, and the overall attack rate was $18.6 \%$. As a follow-up to the outbreak, we investigated whether the administration of a third dose of the MMR vaccine had been protective by stratifying our data according to the timing of vaccination. In line with the report by Cardemil et al. (Sept. 7 issue), ${ }^{1}$ the attack rate was $86 \%$ among the soldiers who had received no vaccination, $15.4 \%$ among those who had received two vaccine doses, and $8.3 \%$ among those who had received three vaccine doses (Table S1 in the Supplementary Appendix, available with the full text of this letter at NEJM.org). Surprisingly, no mumps cases were reported among the soldiers who had received the third MMR dose in the year of the outbreak, but the attack rate was $14.3 \%$ among those who had received the third dose in 2015 or 2016. Although the difference in the attack rates was not statistically significant, this finding may indicate that the timing of the administration of the third MMR dose may be important. Even among persons who have received more than two doses of MMR vaccine, the timely administration of an additional dose may be considered in an outbreak situation.

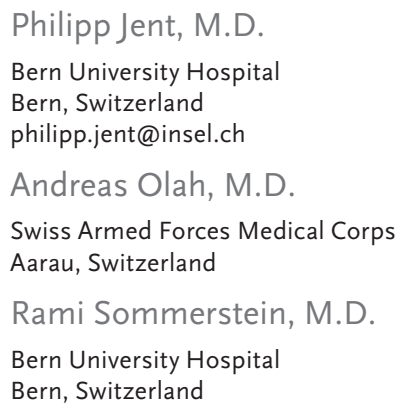

No potential conflict of interest relevant to this letter was reported.

1. Cardemil CV, Dahl RM, James L, et al. Effectiveness of a third dose of MMR vaccine for mumps outbreak control. N Engl J Med 2017;377:947-56.

DOI: 10.1056/NEJMc1714219

TO THE EDITOR: Cardemil and colleagues report a university mumps outbreak that was effectively mitigated with a third dose of the MMR vaccine. The interval since the receipt of the second dose of MMR vaccine was related to the vulnerability of the mumps virus. As compared with students who had received the second dose of the MMR vaccine within the previous 2 years, those who had received it 13 to 15 years earlier had 7 times the risk of infection, and those who had received it 16 to 23 years earlier had 11 times the risk.

The increased attack rate among the students who had been vaccinated at least 13 years before the outbreak corresponds to findings from our recent retrospective epidemiologic assessment involving 3811 military recruits. We found a nonlinear decline in mumps seropositivity over time, with a sharp decrease 13 years after the most recent MMR vaccine dose on the basis of antibody titers obtained at the outset of military training with the use of the BioPlex 2200 MMRV IgG multiplex flow immunoassay (Bio-Rad). Seropositivity fell below the estimated herd-immunity threshold among those last vaccinated at least 16 years earlier (Fig. S1 in the Supplementary Appendix, available with the full text of this letter at NEJM.org). Since recent mumps outbreaks have occurred in populations that had been vaccinated as children, ${ }^{1}$ the findings by Cardemil et al. along with our data raise concern about the need for routine administration of MMR vaccine in young adults entering congregated settings, such as colleges and military training. 
Bryant J. Webber, M.D., M.P.H.

Joshua R. Duncan, M.D., M.P.H.

Uniformed Services University

Bethesda, MD

bryant.webber@us.af.mil

Amy A. Costello, M.D., M.P.H.

U.S. Air Force Academy

Colorado Springs, CO

No potential conflict of interest relevant to this letter was reported.

1. McLean HQ, Fiebelkorn AP, Temte JL, Wallace GS. Prevention of measles, rubella, congenital rubella syndrome, and mumps, 2013: summary recommendations of the Advisory Committee on Immunization Practices (ACIP). MMWR Recomm Rep 2013;62(RR-04):1-34.

DOI: $10.1056 /$ NEJMc1714219

TO THE EDITOR: Cardemil et al. report that a third dose of the MMR vaccine offered protection to previously vaccinated persons in whom immunity had waned. Although the administration of a third vaccine dose may serve as a response to localized outbreaks in universities, one may question whether it is a solution to the overall problem of mumps immunity. An inherent problem with the Jeryl Lynn strain of the mumps vaccine is that it is a different genotype from the mumps strains now circulating. Thus, as antibodies against it wane, they may no longer pro- tect against circulating strains. ${ }^{1}$ It may be technically feasible to mutate Jeryl Lynn to a type G genotype while maintaining its attenuating mutations, and this should be considered. ${ }^{2}$ Another possibility is to develop an inactivated mumps vaccine based on a type $G$ strain, which might give a better boost to vaccinees than a live virus that depends on replication to generate immunity. A killed mumps vaccine was developed in the 1940 s, so it is certainly possible. ${ }^{3}$ When outbreaks of mumps occur among vaccinees, it causes complications and decreases confidence in vaccination.

Stanley A. Plotkin, M.D.

Vaxconsult

Doylestown, PA

stanley.plotkin@vaxconsult.com

Dr. Plotkin reports receiving consulting fees from Merck and GlaxoSmithKline. No other potential conflict of interest relevant to this letter was reported.

1. Gouma S, Ten Hulscher HI, Schurink-van 't Klooster TM, et al. Mumps-specific cross-neutralization by MMR vaccine-induced antibodies predicts protection against mumps virus infection. Vaccine 2016;34:4166-71.

2. Xu P, Chen $Z$, Phan S, Pickar A, He B. Immunogenicity of novel mumps vaccine candidates generated by genetic modification. J Virol 2014;88:2600-10.

3. Stokes J, Enders JF, Maris EP, Kane LW. Immunity in mumps: VI. Experiments on the vaccination of human beings with formolized mumps virus. J Exp Med 1946;84:407-28.

DOI: $10.1056 /$ NEJMc1714219

\section{Molecular Drug-Susceptibility Test for Tuberculosis}

TO THE EDITOR: Xie et al. (Sept. 14 issue) ${ }^{1}$ present the evaluation of a new molecular test for the detection of resistance of Mycobacterium tuberculosis to fluoroquinolones, aminoglycosides, and isoniazid. In the Discussion section, the authors mention that one of the limitations of their study is that the geographic representation is limited to China and South Korea. The geographic origin of a strain of M. tuberculosis is of particular importance for the interpretation of genotypic testing for drug resistance. For example, it has been shown that strains from the Congo area can be erroneously interpreted as fluoroquinolone-resistant with the GenoType MTBDRsl assay (Hain Lifescience) because of local diffusion of a clone carrying an A90G polymorphism in gyrA. ${ }^{2}$ For other antibiotics, variations that are related to geographic location have also been well de- scribed..$^{3-6}$ Thus, the performance of a new genotypic test cannot be extrapolated from one setting to another but needs to be validated in each geographic setting.

Thomas Maitre, M.D.

Hôpital Tenon

Paris, France

thomas.maitre@aphp.fr

Alexandra Aubry, M.D., Ph.D.

Nicolas Veziris, M.D., Ph.D.

Centre d'Immunologie et des Maladies Infectieuses Paris, France

No potential conflict of interest relevant to this letter was reported.

1. Xie YL, Chakravorty S, Armstrong DT, et al. Evaluation of a rapid molecular drug-susceptibility test for tuberculosis. N Engl J Med 2017;377:1043-54.

2. Aubry A, Sougakoff W, Bodzongo P, et al. First evaluation of drug-resistant Mycobacterium tuberculosis clinical isolates from 